\title{
Transferts dans la zone racinaire
}

\author{
Transfers in the root zone
}

\author{
par R. Calvet
}

Institut National Agronomique Paris-Grignon

Unité de science du sol INRA-Grignon

Transport phenomena in the root zone contribute to transfers which take place in the whole soil-plant-atmosphere system. They are often determinant for the circulation of elements towards and in the atmosphere and also towards surface and underground waters. Several sink and source phenomena control the liquid phase composition in the root zone, and then, play an important role in solute transfer processes. This role is illustrated by some examples.

\section{I $\square$ LA ZONE RACINAIRE : UN LIEU DE TRANSFERTS PRIVILÉGIÉ}

La zone racinaire est la couche de surface du sol explorée par les racines des végétaux. Elle se confond parfois avec le sol lui-même quand son épaisseur est petite. Cette zone reçoit de nombreuses substances et divers déchets d'origine variée. Quel que soit leur intérêt, fertilisant ou xénobiotique, les ions et les molécules traversent cette zone et en disparaissent plus ou moins rapidement. L'ensemble des phénomènes qui se déroulent dans cette zone déterminent

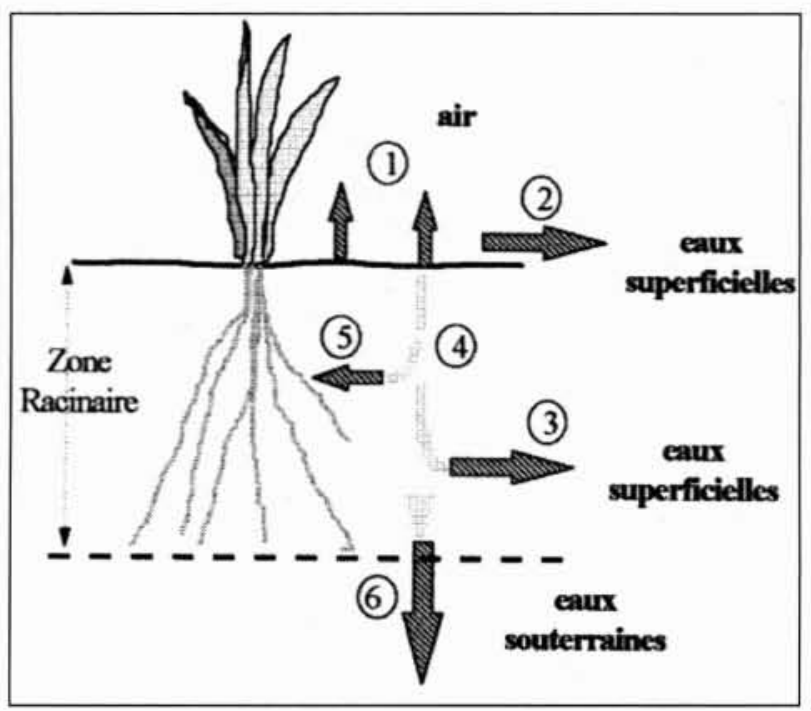

1. Les transferts dans la zone racinaire. l'importance et la nature des transferts :

- vers l'air par volatilisation (1)

- vers les eaux superficielles, soit par ruissellement à la surface du sol (2), soit par écoulement latéral au niveau d'horizons peu perméables (3)

- vers les eaux souterraines par lixiviation (4) et (6)

- dans les plantes par absorption racinaire (5).

L'ensemble de ces transferts met en jeu les phénomènes de diffusion moléculaire et de convection/dispersion.

Les flux de matière dans et hors de cette zone dépendent des modalités de circulation de l'eau et de la composition de la solution du sol, pour ne considérer que la phase liquide. Les concentrations des diverses substances sont régulées par plusieurs phénomènes qui jouent le rôle de puits et de sources. Ces phénomènes ont des caractéristiques très différentes de celles qu'ils présentent en profondeur mais ils concourent de façon déterminante à la rétention plus ou moins réversible et à la dégradation des substances phytosanitaires. Ils sont couplés aux phénomènes de transport de sorte que les transferts dans la zone racinaire en dépendent très étroitement.

L'objectif de cet exposé est de montrer à l'aide de plusieurs exemples comment les phénomènes dans la zone racinaire sont susceptibles, soit de modifier la composition de la solution du sol donc de déterminer les quantités de matière transférées vers les eaux, soit d'induire une structure favorisant les transferts. Les exemples choisis dans le cadre de cet exposé ne concernent que des substances phytosanitaires mais la zone racinaire est tout aussi importante pour les substances fertilisantes et les éléments traces toxiques. 


\section{CARACTÉRISTIQUES GÉNÉRALES DE LA ZONE RACINAIRE}

La zone racinaire se confond en grande partie avec la couche de surface du sol, c'est-à-dire les vingt à quarante premiers centimètres. Des plantes ont des racines plus profondes mais l'essentiel de la biomasse racinaire est localisé dans cette couche. Elle possède des caractéristiques particulières tant sur les plans physique et physicochimique que biologique.

En premier lieu, elle contient des racines qui influencent sa composition et sa structure. Les exudats racinaires sont des substrats facilement métabolisables par la microflore et jouent un rôle dans le devenir des métaux par leurs propriétés complexantes. De plus, les ions excrétés et l'apport de matière organique par les racines mortes font de la zone racinaire une zone où la biomasse microbienne et son activité sont très importantes et les propriétés physico-chimiques du sol différentes de celles d'un sol sans végétation. La figure 2 donne un exemple de la variation de la biomasse microbienne en fonction de la profondeur.

En second lieu, la couche de surface du sol reçoit des apports de substances fertilisantes et phytosanitaires ainsi que des amendements et des déchets liquides et solides variés. Il en résulte un enrichissement en matière organique et en nombreux ions et molécules inorganiques et organiques qui ont une influence à la fois sur les propriétés du sol, notamment sur les propriétés de rétention, et sur l'activité microbienne.

Enfin, la couche de surface du sol est aussi soumise à d'importantes variations de ses caractéristiques physiques. Les passages des outils de travail du sol et la circulation des machines modifient la structure du sol par fragmentation ou par tassement, ce qui a des conséquences sur la circulation de l'eau et des substances dissoutes. En outre, elle est directement soumise aux précipitations et aux fluctuations de la température de l'air, ce qui influence à la fois l'activité microbienne et les transferts par les variations de la structure et de l'état hydrique du sol.

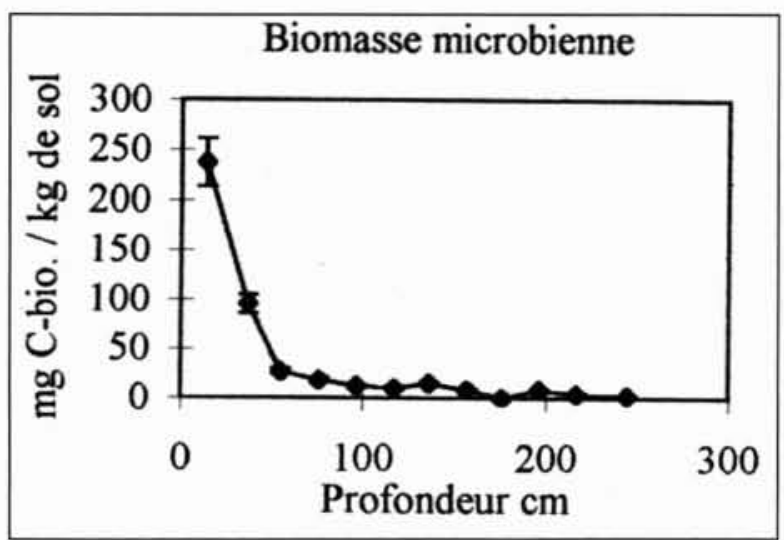

2. Variation de la biomasse microbienne en fonction de la profondeur.

La biomasse est estimée par la méthode de fumigation/extraction. Ces mesures ont été effectuées sur des échantillons prélevés durant l'été 1992 dans un sol cultivé situé à Citeaux près de Dijon. (d'après [1]).

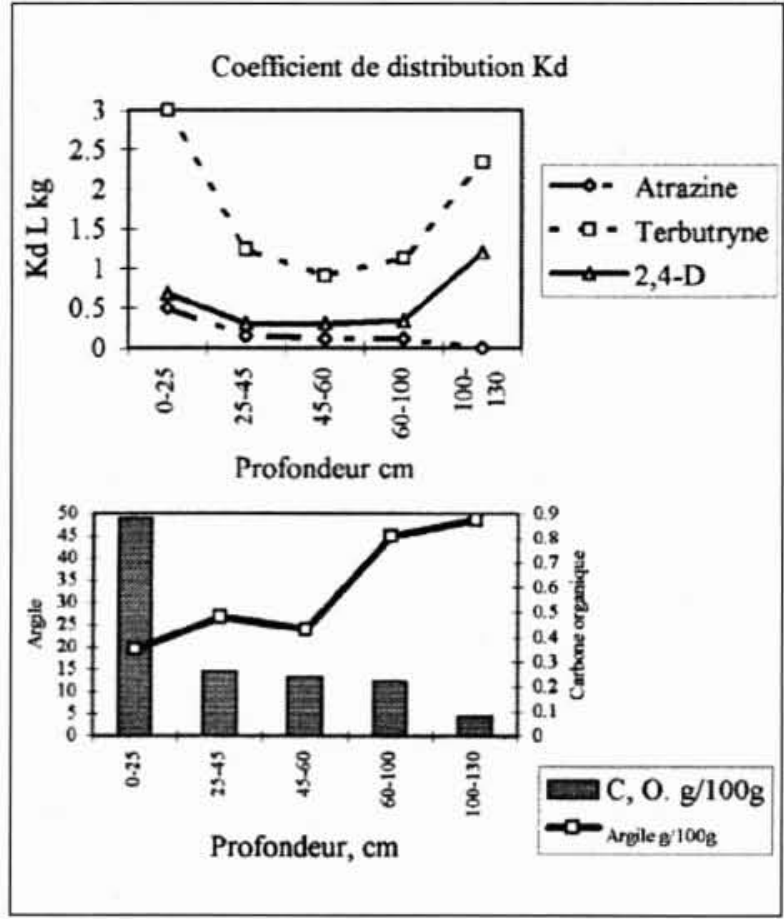

3. Résultats obtenus avec des échantillons de terre prélevés dans un sol cultivé (Rambouillet) [3].

Les courbes représentent les variations du coefficient de distribution $\mathrm{Kd}$ de trois herbicides. II faut rappeler que ces coefficients ne caractérisent correctement l'adsorption que dans le cas où les isothermes d'adsorption sont linéaires ou voisines de la linéarité. Les variations correspondantes des teneurs en argile et en matière organique sont indiquées. Les horizons profonds contiennent des oxydes et hydroxydes de fer. (d'après [3]).

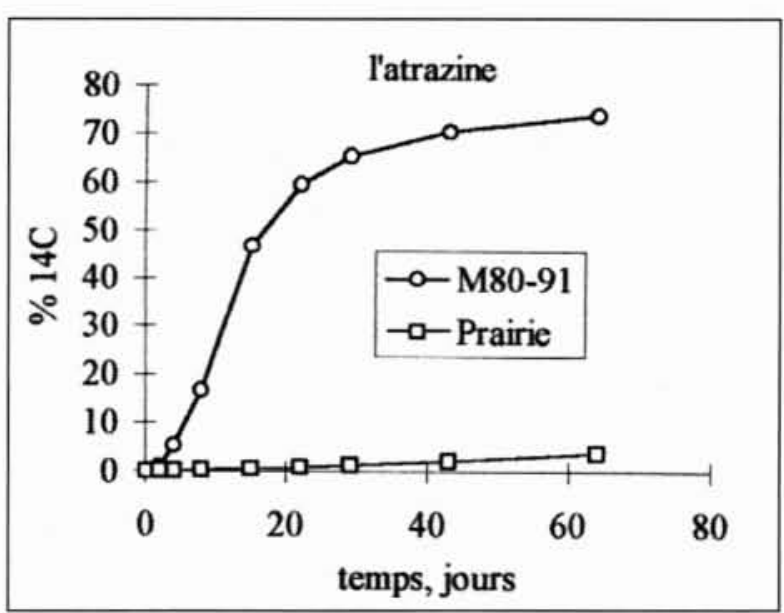

4. Cinétiques de minéralisation de l'atrazine [4].

Elle représente la variation temporelle de la quantité de ${ }^{14} \mathrm{CO}_{2}$ dégagée par la minéralisation des molécules d'atrazine marquées par ${ }^{14} \mathrm{C}$ sur le noyau triazinique. (d'après [4]) 


\section{III — RÉTENTION DES SUBSTANCES PHYTOSANITAIRES}

De très nombreux travaux ont montré le rôle des phénomènes de rétention dans les transferts des substances phytosanitaires en raison de leurs fonctions de puits ou de sources. Ces deux fonctions se manifestent de manière différente car la rétention est rarement réversible et la libération des substances phytosanitaires dans la solution du sol est le plus souvent lente et parfois impossible. La rétention des molécules non ionisées est principalement due à la matière organique et à un moindre degré aux minéraux argileux. En revanche, la rétention des ions est également due à l'ensemble du complexe adsorbant du sol. Toutefois, les minéraux argileux (argiles minéralogiques, oxydes et hydroxydes) présents dans les horizons profonds sont parfois susceptibles d'exercer une rétention importante à l'égard des molécules ionisées [2]. Cela explique pourquoi la rétention de la plupart des substances phytosanitaires non ionisées est généralement plus grande dans la couche de surface. La figure 3 représentant la variation du coefficient de distribution $K d$ de trois herbicides dans un sol cultivé illustre ces considérations.

La matière organique est déterminante pour la rétention d'une molécule non ionisée comme l'atrazine. En revanche, le comportement de la terbutryne est expliqué par l'existence de molécules non ionisées et ionisées sous forme cationique. Quant au 2,4-D, ce sont les oxydes et hydroxydes de fer présents en profondeur qui sont responsables de la rétention, essentiellement par complexation de surface.

Les transferts dans la zone racinaire sont très dépendants de la rétention. Pour les molécules non ionisées, ils sont en général limités par la matière organique qui permet ainsi à la zone racinaire de réduire considérablement les transferts. Il ne faut toutefois pas considérer que la rétention soit toujours négligeable en profondeur.

\section{IV — DÉGRADATION DANS LA COUCHE DE SURFACE}

La dégradation des substances phytosanitaires est due en grande partie à la microflore du sol et, par conséquent, se déroule principalement dans la couche de surface du sol. Elle est influencée par de nombreux facteurs, pédologiques, agronomiques et climatiques, qui tous, ont une action sur les populations microbiennes du sol. L'activité dégradante dépend de la température et de la teneur en eau du sol mais elle dépend également de la nature des populations microbiennes. C'est ce deuxième aspect qui est illustré par les deux exemples suivants. La raison de ce choix est double. $\mathrm{La}$ zone racinaire est très sensible à ces deux facteurs car elle est directement exposée aux actions des variations climatiques et des travaux culturaux. Par ailleurs, la dégradation est classiquement étudiée au laboratoire dans des conditions contrôlées et il faut être conscient de la grande variabilité de ces facteurs qui rend difficile l'utilisation des résultats obtenus.

\subsection{Influence de l'histoire culturale sur la vitesse de dégradation de l'atrazine}

Elle est illustrée par une étude réalisée sur le dispositif expérimental de Grignon où des parcelles ont été diversement cultivées depuis de nombreuses années.

La figure 4 représente les cinétiques de minéralisation de l'atrazine obtenues au laboratoire avec des échantillons de terre prélevés, d'une part dans une parcelle qui a été cultivée en maïs de 1980 à 1991 puis maintenue sans culture jusqu'au moment de l'étude en 1995, d'autre part dans une parcelle portant une prairie. Les deux parcelles ont des caractéristiques pédologiques et climatiques identiques mais diffèrent parce que la première a reçu de l'atrazine pendant 11 années alors que la deuxième n'en a jamais reçu. La très grande différence de vitesse que l'on observe est due à l'adaptation de la microflore à la dégradation de l'atrazine par minéralisation dans un sol qui a reçu cet herbicide pendant plusieurs années. Cette adaptation se traduit également par des modifications importantes du devenir dans le sol de l'atrazine et de ses métabolites comme le montre la figure 5 .

On voit en particulier qu'avec la microflore du sol de prairie, c'est-à-dire non adaptée, la quantité de résidus stabilisés (non extractibles) est considérablement accrue. Parallèlement, la minéralisation et donc l'élimination complète de l'herbicide est au contraire très limitée. Les deux situations présentées sont extrêmes à certains égards mais elles permettent d'attirer l'attention sur la nécessité de prendre en compte l'histoire culturale par les historiques des traitements phytosanitaires. Il apparaît ainsi que la nature et la quantité des résidus peuvent être très dépendantes de l'histoire culturale et par voie de conséquence, les transferts aussi.
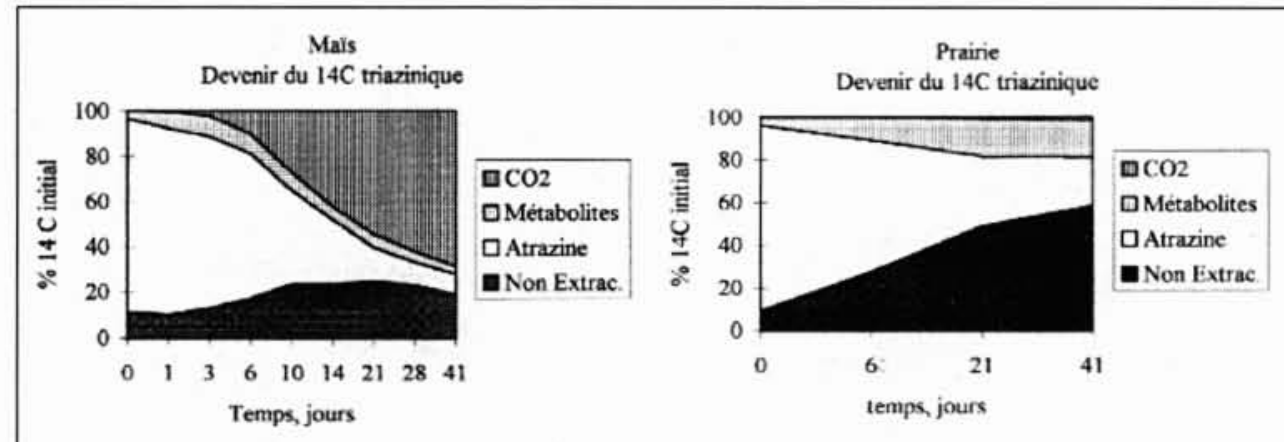

5. Devenir de l'atrazine.

Les quantités de ${ }^{14} \mathrm{C}$ correspondant à l'atrazine et à ses métabolites ont été obtenues par extraction au méthanol. Les quantités dites non extractibles correspondent au ${ }^{14} \mathrm{C}$ qui n'est pas extrait par le méthanol. Toutes les quantités de ${ }^{14} \mathrm{C}$ sont exprimées en $\%$ de la quantité initialement apportée [4]. 


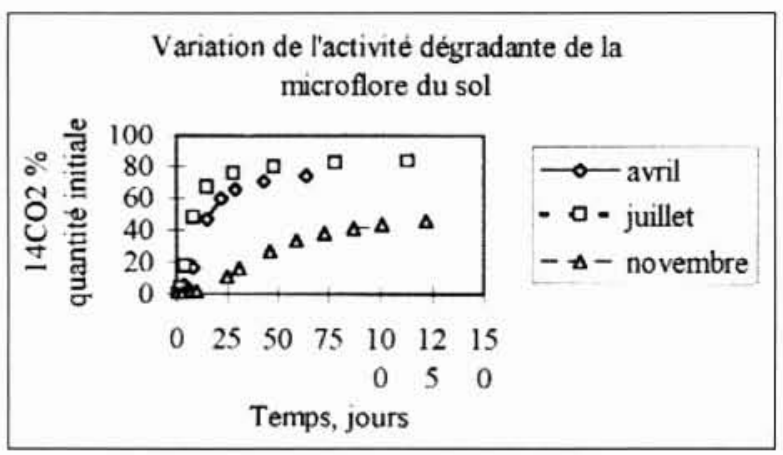

6. Cinétiques de minéralisation de l'atrazine [4].

Les échantillons de terre ont été prélevés à 3 dates différentes et placés dans des conditions contrôlées $\left(28^{\circ} \mathrm{C}\right.$ et $80 \%$ de la capacité de rétention en eau du sol) (d'après [4]).

\subsection{Influence des conditions climatiques sur les propriétés dégradantes de la microflore}

Les caractéristiques de la microflore du sol varient en fonction des conditions climatiques. Une façon de mettre en évidence cette variation consiste à déterminer l'aptitude à la dégradation de la microflore contenue dans des échantillons de terre prélevés dans un sol à différents moments dans l'année. La figure 6 en donne un exemple.

Ces deux exemples montrent la grande sensibilité de la microflore du sol à l'égard de l'histoire culturale et des conditions climatiques. Cela signifie que l'activité dégradante de la microflore du sol peut être très variable au cours de l'année en un lieu donné ; la persistance des substances phytosanitaires pourra également être très variable. De plus, la caractérisation de cette activité ne peut pas être faite simplement sur la base d'une seule valeur de la vitesse de minéralisation déterminée sur un échantillon quelle que soit sa date de prélèvement.

\section{V $\square$ TRANSPORT DANS LA ZONE RACINAIRE}

Compte tenu de ce que l'on sait sur la zone racinaire, donc la couche de surface du sol, on peut penser que les transferts y présentent des caractéristiques différentes par rapport aux zones plus profondes. Parmi ces particularités, il faut citer l'existence d'une importante rétention due à l'accumulation des matières organiques et les modifications de la structure du sol provenant entre autres des actions du climat.

\section{- 5.1 Influence de la présence de racines}

Un sol enherbé possède une couche de surface où les racines sont très denses et la teneur en matière organique élevée. Il en résulte des transferts modifiés par rapport à un sol cultivé en raison d'une activité microbienne et d'une rétention différentes. Cela est illustré par la figure 7. Elle montre des résultats obtenus sur des colonnes de sol non perturbé prélevées dans deux parcelles, l'une enherbée, l'autre cultivée. La molécule étudiée est l'isoproturon qui a été apporté sur les colonnes avec un marquage au ${ }^{14} \mathrm{C}$. Les colonnes ont été soumises à une percolation par de l'eau pendant 15 jours et ensuite découpées pour être analysées. La figure 7 représente les répartitions des quantités d'isoproturon extraites par l'eau et le méthanol ainsi que des quantités non extractibles. Par ailleurs, les mesures montrent que la quantité d'isoproturon lixiviée est plus grande dans le sol cultivé $(47 \pm 5 \%)$ que dans le sol enherbé $(30 \pm 5 \%)$. On constate ainsi que l'enherbement entraîne une rétention de I'herbicide par la formation de résidus stabilisés (non extractibles par le méthanol) et limite le transfert en profondeur. II n'est cependant pas sans intérêt de s'interroger sur le devenir des résidus stockés dans les premiers centimètres de la couche de surface.

\subsection{Influence de la structure du sol sur les transferts}

La couche de surface du sol est soumise à des variations importantes de structure du fait des diverses actions mécaniques qu elle subit mais aussi en raison des variations climatiques qui modifient la teneur en eau du sol et sa structure. Il en résulte une hétérogénéité plus ou moins

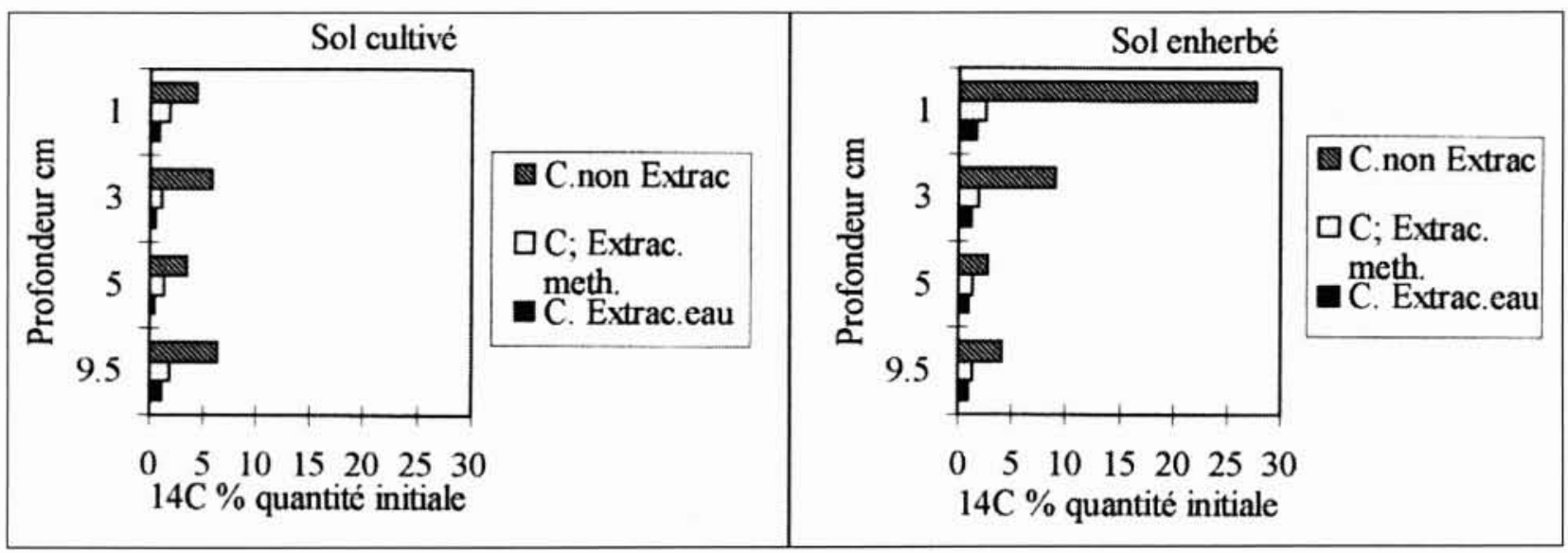

7. Influence d'un enherbement sur la distribution du ${ }^{14} \mathrm{C}$ apporté sous forme d'isoproturon marqué (d'après [5]). 


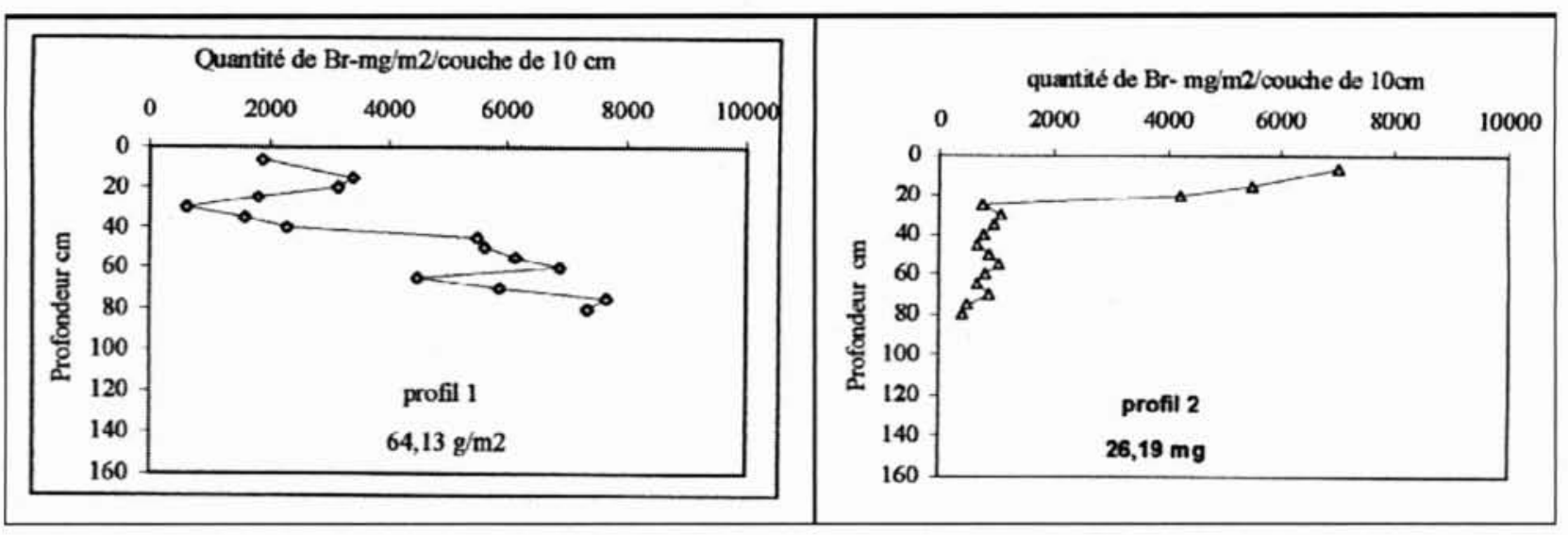

8. Transferts préférentiels [6] révélés par deux profils de distribution de l'anion bromure situés à $1,2 \mathrm{~m}$ l'un de l'autre observés sur le site de Villamblain (d'après [6])

grande qui se traduit par l'existence de transferts préférentiels comme le montre la figure 8 qui représente des profils de distribution de l'anion bromure dans un sol.

L'application du bromure a été effectuée le 29-01-1996 à la dose de $80 \mathrm{~g} / \mathrm{m}^{2}$ et les mesures 44 jours après. Les quantités retrouvées dans les quatre-vingts premiers centimètres sont de $64,13 \mathrm{~g} / \mathrm{m}^{2}$ et $26,19 \mathrm{~g} / \mathrm{m}^{2}$ pour les profils 1 et 2 respectivement. Compte tenu du volume de pluie reçu par le sol entre l'application et les prélèvements, on aurait dû trouver une distribution du bromure centrée aux alentours de $20-30 \mathrm{~cm}$ de profondeur. Les allures très différentes des distributions observées et les quantités retrouvées inférieures aux quantités apportées indiquent l'existence d'un transport préférentiel important en profondeur et très variable dans l'espace. On peut donc penser qu'un certain transfert en profondeur de substances phytosanitaires est également possible.

\section{VI $\square$ CONCLUSION}

Les quelques exemples qui viennent d'être donnés et discutés montrent que les transferts dans la zone racinaire sont complexes. Leur description et leur compréhension imposent de connaître convenablement les phénomènes puits et sources. Il faut insister sur le fait que cette zone est caractérisée par des phénomènes physico-chimiques et microbiologiques intenses dont les variabilités spatiales et temporelles sont grandes.

Par la rétention due à la matière organique, la zone racinaire limite beaucoup le transport des substances phytosanitaires qui sont souvent localisées dans les 10 à 30 premiers centimètres du sol. Cet aspect positif s'accompagne cependant d'une interrogation quant aux résidus liés. En effet, leur nature et leur devenir sont encore très mal connus, de sorte que la rétention observée n'est pas complètement décrite. La dégradation dans la zone racinaire est intense en raison de l'importante activité de la microflore mais des questions demeurent au sujet de sa très grande sensibilité aux interventions culturales et aux variations climatiques dont les conséquences sont aujourd'hui difficiles à prévoir. La rétention et la dégradation font que la zone racinaire exerce une action de protection à l'égard des eaux et se comporte comme un filtre bio-physicochimique. Toutefois, ce filtre présente des défauts et des transferts en profondeur sont possibles grâce à des voies de circulation préférentielles comme les fissures et les canalicules biologiques. On ne connaît pas leur réelle importance et il sera très utile de mieux comprendre leur déterminisme et leur fonctionnement hydrodynamique.

Toutes ces considérations attirent l'attention sur notre capacité aujourd'hui très limitée à décrire les transferts dans la zone racinaire. Cela explique pourquoi il est difficile, voire impossible, de simuler correctement le devenir des substances phytosanitaires dans les sols. Cela conduit à inviter à la plus grande prudence dans la mise en œuvre des divers modèles numériques dont l'usage tend à se développer.

\section{Références}

[1] Dictor M.C., L. Tessifr and G. SOULAs, 1998. - Reassessment of the Kec coefficient of the fumigation-extraction method in a soil profile. Soil Biol. Biochem., 30 (2), 119-127.

[2] CAL.VET R., 1989. - Adsorption of organic chemicals in soils. Environmental Health Perspectives, 83, 145-177.

[3] Barriuso E., 1995. - Etude de l'adsorption des herbicides sur les sols de la région de Rambouillet. Rapport interne, Unité de science du sol de Grignon, France.

[4] BARriuso E. and S. Houot. 1996. - Rapid mineralization of the s-triazine ring of atrazine in soils in relation to soil management. Soil Biol. Biochem., 28 (10/11), 1341-1348.

[5] Vidon P., 1997. - Rôle des matières organiques des sols des bandes enherbées dans la dégradation et la rétention des pesticides : cas de l'isoproturon. Mémoire de fin d'études du DEA * Fonctionnement physique. chimique et biologique de la biosphère continentale ". Université Paris VI, INA-PG, ENS.

[6] VACHIER P. ET A. BRUAND, 1996. - Etudes des transferts préférentiels sur le site de Villamblain ; conséquences pour le transfert des produits phytosanitaires. Rapport d'étape d'une action de recherche GREPPES. INRA. Unités de science du sol d'Orléans et de Grignon. 